\title{
ERRATUM
}

\section{Pyrazine Carboxylamidase Activity in Corynebacterium}

IOAN T. SULEA, MARJORIE C. POLLICE, AND LANE BARKSDALE

Department of Microbiology, New York University School of Medicine and Medical Center, New York, New York 10016

Vol. 30, no. 2, p. 467, Table 1: C. diphtheriae strains Lorente and $\mathrm{PW} 8_{\mathrm{r}}(\mathrm{P}) \mathrm{Tox}^{+}$should have been listed under $C$. diphtheriae ( intermedius) rather than under $C$. diphtheriae (mitis). 